\title{
Lingual frenectomy with diode high-power laser in an adult patient: a case report
}

\begin{abstract}
Ankyloglossia or tongue-tie is a congenital dysfunction, characterized by an abnormality of the tongue frenulum, where it can be adhered in a short form to the mouth floor or too close to the tip of the tongue, of fibrous appearance. The indication of surgical removal of the lingual frenulum occurs when the movements of the tongue and the mastication, swallowing and phonation functions are significantly altered. The surgical removal of this frenulum-frenectomy is the best option to solve this condition. The surgical technique can be executed in a conventional form or by laser, presenting differences on healing and postoperative period. Female patient, 20years old, sought the extension project "Laser Therapy in Dentistry" of the Federal University of Maranhão relating swallowing and phonation difficulties. During the clinical exam it was asked to the patient to protrude her tongue, which resulted in restrictions of its functions and movements. The lingual frenectomy was then made with high-power diode laser. At the end of the surgery it was also performed the protocol of tissue repair with low-power laser, following the parameters: $660 \mathrm{~nm}, 100 \mathrm{~mW}, 3 \mathrm{~J}$ of energy per point for 30 seconds in each point. 6 points were irradiated in the borders of the surgery incision to accelerate healing, in a total of 3 sessions. The patient did not relate any pain or edema after surgery. Therefore, the use of high-power laser associated to low-power laser was effective in the treatment of ankyloglossia, reduction of edema and postoperative healing.
\end{abstract}

Keywords: frenectomy, ankyloglossia, swallowing difficulties, laser therapy in dentistry, stomatognatic system
Volume 2 Issue I - 2018

\section{Guilherme Silva Furtado,Winnie Dandara Rocha Ferreira,Amanda Ferreira de Sousa Pinheiro,Andréa Dias Neves Lago}

Department Odontology I, University Federal of Maranhão, Brazil

Correspondence: Andréa Dias Neves Lago, Department of Odontology I, Federal University of Maranhão (UFMA), Av dos Portugueses 1966, Bacanga, São Luís, MA, Zip Code 65080-805, Brazil,Tel +55 98 3272-8570, 98-9197-14II,

Email adnlago@gmail.com

Received: November 24, 2017 | Published: February 09, 2018

\section{Introduction}

Ankyloglossia or tongue-tie is a congenital dysfunction, characterized by abnormality of the lingual frenulum, where it can be adhered in a short form to the mouth floor or too close to the tip of the tongue generating difficulties to individuals. ${ }^{1-4}$ The lingual frenulum is a normal anatomic structure characterized by being a median fold that connects the tongue to the mouth floor; is exclusively constituted by fibrous dense conjunctive tissue, besides superior fibers of the genioglossus muscle. ${ }^{2,5}$ However, this structure is dynamic and modifiable, susceptible of changes on its shape, size and/or insertion during its embryonic formation. ${ }^{2,3}$

This abnormality has genetic etiology and/or environmental factor, being able to reach individuals of both sexes and any age. ${ }^{1,3}$ Clinically it presents painful symptomatology, movement, phonation and sometimes swallowing difficulties, ${ }^{1-3}$ being indicated the surgical removal. ${ }^{1,6}$ During clinical exam is recommended to ask the patient to do a protrusion movement to evaluate the extension reached by the tongue.

The frenectomy is the best option to solve this condition, where the frenulum is reinserted in a correct form during its healing after the surgical procedure. ${ }^{2}$ The surgical technique can be executed in a conventional form or by laser, presenting differences in the execution, healing and postoperative period. ${ }^{6}$ The conventional frenectomy is a simple procedure that consists in the use of scissors and scalpels for cutting or section of the lingual frenulum and posterior suture for first intention healing. The use of high-power laser is indicated of frenectomy because of its exclusive interaction with the exposed tissue due to its wavelength and specific active medium, besides its secondary therapeutic effects that proportionate a more efficient healing and a better postoperative period to the patient. ${ }^{1,6}$

The objective of this study is to report a case of lingual frenectomy with high-power laser and its association with diode low-power laser on postoperative period.

\section{Case report}

Female patient, 20 years old, sought the extension project "Laser therapy in Dentistry" at Federal University of Maranhão relating swallowing difficulties and phonation problems. During the clinical exam it was asked to the patient to perform protrusion and laterality movements of her tongue, where it was noticed restrictions on its functions and movements (Figure 1). The base and tip of the tongue insertion made the adequate protrusion difficult and occasioned a "V" fold on it (Figure 2).

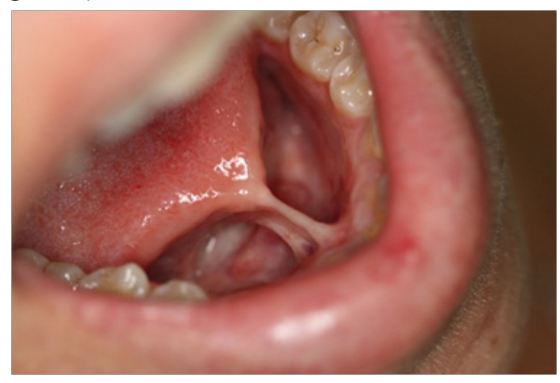

Figure I Initial. 


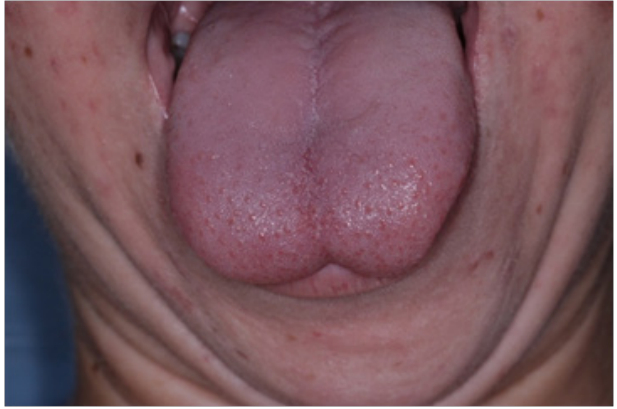

Figure 2 Initial- Observe tongue limitation.

This condition caused to the patient difficulties on swallowing and phonation associated to the limitations of the lingual movements. It was opted to perform the lingual frenectomy with diode high-power laser (Figure 3) due to its benefits described in literature. The procedure was performed by the diodo high power laser (Thera Lase Surgery, DMC, São Carlos, SP, Brazil). The settings used were power output $2 \mathrm{~W}$, a continuous wavelenght of $880 \mathrm{~nm}$ in infrared emission; the beam was delivery by a $300 \mu \mathrm{m}$ optical fiber; energy level $120 \mathrm{~J} ; 20 \mathrm{pps}$.

After frenectomy was completed, the photobiomodulation terapy following the parameters: $660 \mathrm{~nm}, 100 \mathrm{~mW}, 3 \mathrm{~J}$ of energy per point, for 30 seconds on each point. 6 points were irradiated on the borders of the surgery incision to accelerate the healing, in a total of 3 sessions (Figure 4). Before performing the surgery, the patient received a small amount of anesthetic that was injected with a short needle into the region around the lingual frenulum.

After 14 days the operated area was totally healed and the patient did not present any pain or edema during the healing process. The fact suggested an excellent prognosis to the case (Figure 5).

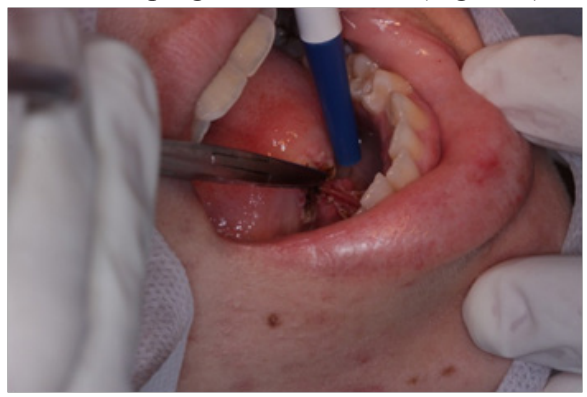

Figure 3 During surgery.

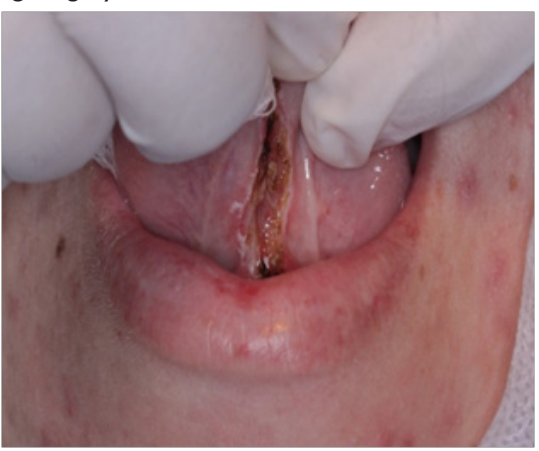

Figure 4 Immediately after the frenectomy.

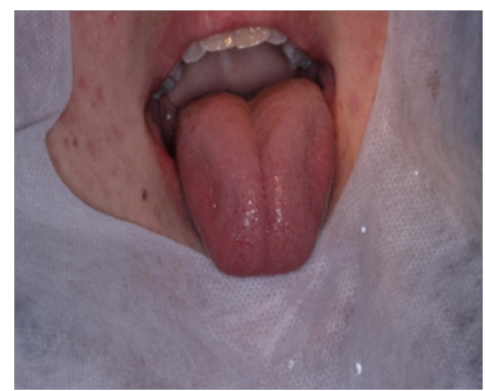

Figure 5 After 14 days the operated area was totally healed.

\section{Discussion}

The lingual frenectomy, independent of technique, has its efficacy proved by several studies. The tongue is an organ of major importance in the stomatognatic system, possessing vital functions such as swallowing food, influence in the teeth position in the dental arches and an important psychosocial paper, for it is essential in the pronunciation of words. An anomalous lingual frenulum harms the tongue anatomical and physiological functions, restricting its movements.

In the case here described, the frenulum inserted too close to the tip of tongue presented important relevance, so that it caused morphofunctional dysfunctions to the patient. The main complaint was difficulties in pronouncing certain words and an altered physiological pattern of swallowing due to the abnormal frenulum, besides limitations in amplitude of tongue movements

The development of new technologies that make a safe and efficient treatment to the patient possible, with a better prognosis, is of major importance. This way, the system here presented on this case report has as its major advantage the absence of discomfort during and after the surgical procedure in comparison to the conventional method. The absence of bleeding, pain or edema is a positive factor in the surgical procedure performed with high-power diode laser, because it has exclusive affinity to the tissue in contact, besides secondary biological effects that assist in the postoperative period ${ }^{6}$.

The association of the surgical procedure made with highpower laser and the application of low-power laser on infrared wavelength has its efficacy scientifically proved. Low-power laser has biomodulatory effects, acting directly in the cellular mitochondria, accelerating cellular metabolism and consequently generating benefits to individuals. ${ }^{6}$ Low-power laser used in adequate parameters assists on healing process and inhibits the appearance of pain or edema. ${ }^{6}$

It was possible to verify, in this case report, that the lingual frenectomy was effective, once the lingual frenulum healed after 14 days, reinserting itself in the possible correct anatomic position. The patient did not relate any pain or edema in any moment after surgical procedure, thus also demonstrating the low-power laser effectiveness.

\section{Conclusion}

The use of high-power diode laser for surgical procedures such as lingual frenectomy and the use of low-power laser as complementary therapy is already an innovative reality and a great advance to modern dentistry, since the possibility of performing a conservative surgical 
procedure, with absence of pain and postoperative edema, returning the correct stomatognatic functions to the patient is of major relevance.

\section{Acknowledgements}

None.

\section{Conflicts of interest}

The author declares no conflict of interest.

\section{References}

1. Junqueira MA, Cunha NN, Costa e Silva LL, et al. Surgical techniques for the treatment of ankyloglossia in children: a case series. J Appl Oral Sci. 2014;22(3):241-248.

2. Khan MA, Farooqui M, Saimbi CS, et al. Untangle the lingual aberrant frenum by diode laser: A case report with review of literature. $J$ Dent Lasers. 2016;10:34-36.
3. Bahadure RN, Jain E, Singh P, et al. Labial ankyloglossia: A rare case report. Contemp Clin Dent. 2016;7(4):555-557.

4. Jangid K, Alexander AJ, Jayakumar ND, et al. Ankyloglossia with cleft lip: A rare case report. J Indian Soc Periodontol. 2015;19(6):690-693.

5. Komori S, Matsumoto K, Matsuo K, et al. Clinical study of laser treatment for frenectomy of pediatric patients. Int J Clin Pediatr Dent. 2017;10(3):272-277.

6. Akpinar A, Toker H, Lektemur Alpan A, et al. Postoperative discomfort after Nd:YAG laser and conventional frenectomy: comparison of both genders. Aust Dent J. 2016;61(1):71-75. 